\title{
Application of Intelligent Technique for Development of Colpitts Oscillator
}

\author{
Mohamad Ghazali b. Ameer Amsa ${ }^{1}$, A. M. Aibinu ${ }^{2}$ and M. J. E. Salami ${ }^{1}$ \\ ${ }^{1}$ Mechatronics Department, Kulliyah of Engineering, \\ International Islamic University Malaysia (IIUM) \\ Gombak, Malaysia. \\ irghazali@yahoo.com \\ ${ }^{2}$ Department of Telecommunication Engineering, \\ Federal University of Technology, \\ Minna, Nigeria \\ maibinu@gmail.com
}

\begin{abstract}
In this paper, new method of Colpitts oscillator designing through combination of Genetic Algorithm and Artificial Neural Network (ANN) has been suggested. The Thevenin's resistors for the common base Colpitts oscillator are optimized through application of GA and ANN. The developed common base Colpitts oscillator has shortest transient time response and stable Direct Curent (DC) stability in the long term operation. Involvement of GA and ANN successfully optimize between transient time response and steady state response of common base oscillator. Application of these two artificial intelligent techniques assist faster selection of optimizes components values such as resistance values during circuit development rather than conventional method which used intuition techniques to develop the circuit.
\end{abstract}

Keywords- Colpitts oscillator; Genetic Algorithm; Artificial Neural Network; Intelligent Technique

\section{INTRODUCTION}

The main purpose of oscillator is to generate particular waveform at certain frequency and amplitude in the consistence way. Oscillators are categorized based on three main characteristics, namely, based on output waveform, circuit component and range of operation frequency [1]. An oscillator can be classified either sinusoidal or non sinusoidal by consider the output waveform produced by that oscillator. As it name suggest, sinusoidal oscillators is used to generate sinusoidal, while non sinusoidal or relaxation oscillator is used to produce non sinusoidal waves such as pulse, triangular, saw tooth waves etc. The classification based on the circuit component distinguishes oscillators into RC oscillators and LC oscillators. To clarify, the oscillators those designed by using combination of resistors and capacitors are known as RC oscillators. On the other hand, oscillators those have inductor and capacitors components are called LC oscillators. The third classification based on range of operation frequency further divides oscillators as low frequency oscillator and high frequency oscillator [1]. Oscillator that generates oscillation frequency around $20 \mathrm{~Hz}$ to $100-200 \mathrm{~Hz}$ are classified as low frequency or audio frequency oscillator, while high frequency oscillator refer to the oscillator which generates output waveform in the radio frequency range around 200 to $300 \mathrm{kHz}$ up to Gigahertz [1]. Traditionally, oscillator designers mostly used analysis or intuition techniques to develop the circuit. In the present day, besides of simulation technique, application of intelligent technique such as Artificial Neural Network (ANN) and evolution algorithm such as genetic algorithm (GA) and Particle Swarm Optimization (PSO) techniques are used for analog circuit optimization [2]-[4]. As the utilization of GA and ANN in the analog circuit modeling increasing, there are few of works lead the application of this method in the oscillator design field. Genetic Algorithm basically used to discover similar behavior circuits with different topology or structure. For example, in [5], hybrid technique through combination of neural networks and genetic algorithm is applied for develop $30 \mathrm{GHz}$ Voltage Controlled Oscillator (VCO) and $60 \mathrm{GHz}$ oscillator. Neural networks is applied for model the circuit, while the parameters are optimized by means of genetic algorithm in order to obtain greater number of possible circuits those meet the desired specification [5]. In [3], genetic algorithm (GA) is utilized to evolve multiple designs of sinusoidal oscillator that use single op amp as amplifier component. Most of the oscillators evolved from this technique conform to analytical result. GA is used to automatically discover oscillator circuits with optimization desired target frequency [6]. Evolutional technique is used to generate a number of oscillators with different structures where fault condition is included in the fitness function [7]. In this research, combination of GA and ANN is used to develop common base Colpitts oscillator.

\section{COLPITTS OSCILLATOR MODELING AND ANALYSIS}

The proposed Colpitts oscillator design is shown in the Fig. 1. It consists of three resistors, two capacitors and one inductor as passive element for this circuit. The feedback network is tapped between two capacitors. The emitter terminal of the Bipolar Junction Transistor (BJT) is connected with the feedback network in order to maintain the oscillation output without aid of external input. 


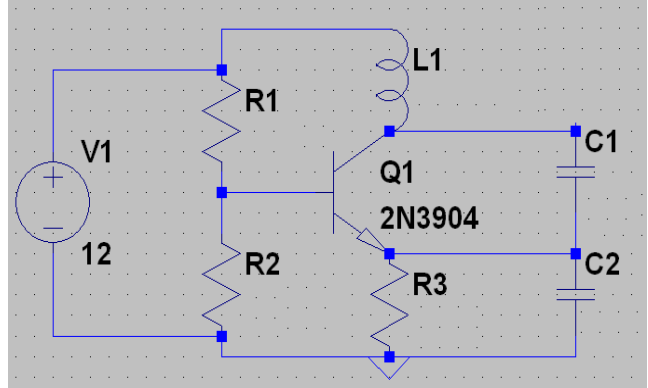

Fig.1. Common base Colpitts oscillator

The oscillation frequency of the oscillator is calculated by using

$$
f=\frac{1}{2 \pi \sqrt{L C_{\text {parallel }}}} ; C_{\text {parallel }}=\frac{C_{1} C_{2}}{C_{1}+C_{2}}
$$

Large signal analysis is used to analyze Direct Current (DC) operating point and collector current, $I_{c}$ of the oscillator. In the large signal analysis, the voltage divider circuit is simplified by using Thévenin's equivalent resistance, $R_{t h}$, and voltage, $V_{t h}$ as in the Fig. 2, where

$$
\begin{gathered}
R_{t h}=R_{1} \| R_{2}=\frac{R_{1} R_{2}}{R_{1}+R_{2}} \\
V_{t h}=\frac{R_{2}}{R_{1}+R_{2}} V_{C C}
\end{gathered}
$$

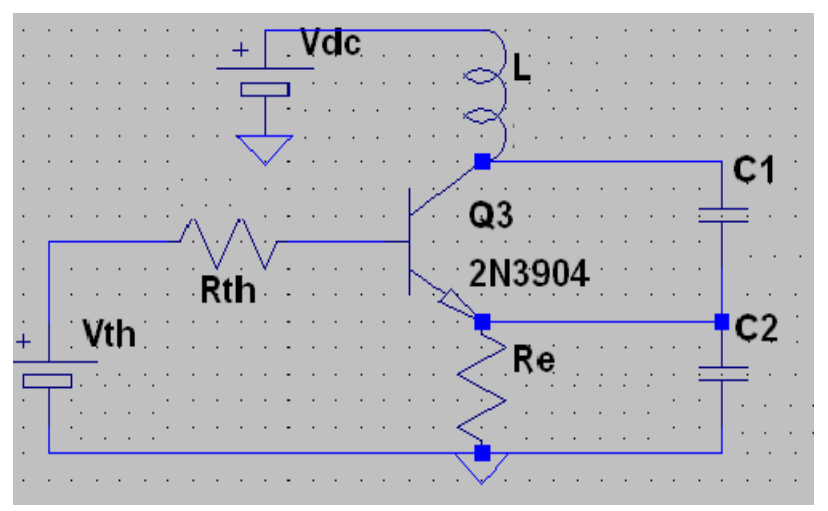

Fig. 2. Thevenin's equivalent of oscillator

Large signal analysis is conducted by replace the capacitors with open circuits and inductance with a short circuit. Fig. 3 shows the forward active region of BJT, where the term $B, C$ and $E$ are refer to base, collector and emitter terminals respectively. The complete large signal analysis model of the oscillator can be developed through combination of Thevenin's equivalent circuit for the bias network (Fig. 2) and large signal analysis of BJT (Fig. 3). The collector current, $I_{c}$ is given by $I_{c}=h_{f e} I_{B}$ and base current is calculated as $I_{B}=\frac{I_{C}}{h_{F E}}$



Fig. 3. BJT Large signal analysis

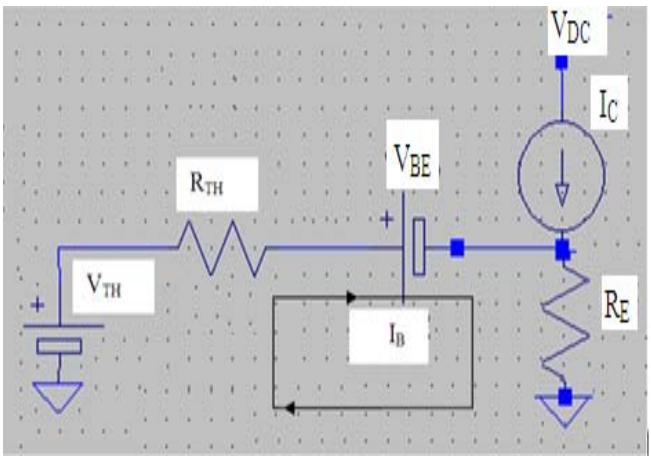

Fig. 4. Large-signal model of oscillator

By applying Kirchhoff's voltage law to the loop in the Fig. 4 gives

$$
-V_{T H}+\frac{I_{c}}{h_{F E}} R_{T H}+V_{B E}+\frac{I_{c}}{h_{F E}} R_{E}+I_{C} R_{E}=0
$$

Rearranging (4) gives

$$
I_{C}=\frac{V_{T H}-V_{B E}}{R_{E}\left(1+\frac{1}{h_{F E}}\right)+\frac{R_{T H}}{h_{F E}}}
$$

By assuming $\frac{1}{h_{F E}}<<1$, (5) becomes

$$
I_{C}=\frac{V_{T H}-V_{B E}}{R_{E}+\frac{R_{T H}}{h_{F E}}}
$$

Generally, BJT has two major parameters comprising of DC current gain $\left(h_{F E}\right)$ and base-to-emitter voltage drop $\left(V_{B E}\right)$ which may change due to temperature fluctuation after long time operation of the circuit. Base on (6), the variation of these two variables may lead to change of $I c$ of the oscillator. Inconsistence of $I c$ will cause instability of DC operating point of the circuit. Thus, the influence of the aforementioned variables should be minimized in order to obtain long term DC operating point stability.

Equation (6) shows the $I c$ value is directly proportional to $V_{T H}$ and $V_{B E}$ (According to datasheet of BJT 2N3604 model the $V_{B E}$ is 0.7 Volt at room temperature). In case the Thévenin's equivalent voltage $\left(V_{T H}\right)$ is too small or very close to $V_{B E}$ 
$(0.7 \mathrm{~V})$, smallest variation of $V_{B E}$ will be significantly changed bias collector current, $I_{c}$. Therefore, the $V_{T H}$ value should be selected with relatively much greater value compare to $V_{B E}$ in order to minimize the influence from variation of $V_{B E}$. On the other hand, the denominator part in (6) shows the significant contribution of the $h_{F E}$ to $I_{c}$ fluctuation can be reduced through properly select emitter resistance $R_{E}$ to be much greater than the ratio of $\frac{R_{T H}}{h_{F E}}$. The $R_{T H}$ is expected to be $100 \mathrm{k} \Omega$ based on assumption $h_{F E}$ around 200 during operation since the expected collector current is around $2 \mathrm{~mA}$. Thus, $R_{E}$ is selected to be $10 \mathrm{k} \Omega$ in order to make it much greater than ratio of $\frac{\mathrm{R}_{\mathrm{TH}}}{\mathrm{h}_{\mathrm{FE}}}$

The objective of oscillator design in this study is to maximize Thevenin's equivalent voltage $\left(V_{T H}\right)$ in order to maintain long term DC voltage stability, while attain shortest transient time response during start up period. The $V_{T H}$ value is directly proportional with the resistors $(R 1$ and $R 2)$ and power supply voltage, $V_{s}$ as shown in (3). Thus, the main concern during the design process is proper selection of $R 1$ and $\mathrm{R} 2$ resistors since it directly determine the $V_{T H}$ of the oscillator circuit. Besides that, these two resistances combination determine the initial amount of input current (before oscillation occurs) supplied to the transistor, since the resistor $R 1$ and $R 2$ are used as voltage divider circuit. In case the supplied current is too low, damped oscillation may occurs which lead to oscillation wave disappears. On the other hand, if the supplied current to the transistor is too high, the oscillator may produce distorted sine wave as an output. Thus, proper selection of $R 1$ and $R 2$ resistance values is necessary since its influence the quality of the sine wave and DC operating point stability of the oscillator.

Simulation is conducted (by using LTspice software) in order to examine since wave output quality that produced by the oscillator for various combination of resistance value (from hundereds-ohm to Mega-ohm). In the first step, large amount of possible combination of resistance values are divided into five ranges of resistor group as shown in the Fig. 5 .



Fig. 5. Resistors simulation range

The simulation objective is to analyze the effect of various combinations of Thévenin's resistors $(R l$ and $R 2)$. The results from these simulations are shown in the Appendix section. The results from the simulations show that the pair of $R l$ and $R 2$ resistors with values in the range of kilo-ohm to Megaohm only were able to produces pure sine waveform for the designed common base Colpitts oscillator in this study (as shown in the Fig. 11). Higher range of resistor pair produces damped oscillation waveform (as simulated in the Fig.12), whereas lower range of resistors pair generates distorted sine wave as output (as shown in Fig. 8, Fig. 9 and Fig. 10). Thus, suitable range of the combination of resistance value is identified from $100 \mathrm{k} \Omega$ to $1 \mathrm{M} \Omega$. Thus, more depth analysis is conducted by using various combinations of $R 1$ and $R 2$ in this range of $100 \mathrm{k} \Omega$ to $1 \mathrm{M} \Omega$ resistance values in order to analysis transient response of the circuit. The time taken by the sine wave to achieve steady amplitude is measured. The results from these simulations are listed in the table I.

TABLE I. TRANSIENT RESPONSE TIME FOR VARIOUS COMBINATIONS OF $R_{l}$ AND $R_{2}$ RESISTORS

\begin{tabular}{|c|c|c|}
\hline $\boldsymbol{R} \boldsymbol{1}(\boldsymbol{\Omega})$ & $\boldsymbol{R} \mathbf{(} \boldsymbol{\Omega})$ & Transient Time (ms) \\
\hline $100 \mathrm{k}$ & $100 \mathrm{k}$ & 2.57 \\
\hline $100 \mathrm{k}$ & $200 \mathrm{k}$ & 2.24 \\
\hline $100 \mathrm{k}$ & $500 \mathrm{k}$ & 0.91 \\
\hline $100 \mathrm{k}$ & $900 \mathrm{k}$ & 1.21 \\
\hline $100 \mathrm{k}$ & $1 \mathrm{M}$ & 1.32 \\
\hline $200 \mathrm{k}$ & $500 \mathrm{k}$ & 1.24 \\
\hline $300 \mathrm{k}$ & $500 \mathrm{k}$ & 1.51 \\
\hline $500 \mathrm{k}$ & $500 \mathrm{k}$ & 1.59 \\
\hline $1 \mathrm{M}$ & $500 \mathrm{k}$ & Oscillation wave disappears \\
\hline $1 \mathrm{M}$ & $1 \mathrm{M}$ & Oscillation wave disappears \\
\hline $1 \mathrm{M}$ & $100 \mathrm{k}$ & 7.2 \\
\hline
\end{tabular}

The results in the Table 1 show the values of resistors $R l$ and $R 2$ are affect the transient response time of the oscillator. Since the resistor of $R 1$ and $R 2$ used as voltage divider circuit, the initial amount of input current (before oscillation occurs) supplied to the transistor is depends on $R 1$ and $R 2$ resistance values. In case the input current is too low $(R 2<<R 1$ or $R 2$ is very high resistance), the damped oscillation may occur which lead to oscillation wave disappears.

The transient response time analysis shows each combination of the resistor lead to different transient response time. At the mean time, the combination of resistors that give maximum $V_{T H}(R l=100 \mathrm{~K} \Omega$ and $R 2=1 \mathrm{M} \Omega)$ does not give lowest transient response time. Thus, in order to achieve the design objective in this research, optimization between transient response time and $V_{T H}$ is required. By using the conventional method, substantial number of simulations is needed to find the transient response time for combination of resistor near to the value of $R 1=100 \mathrm{~K} \Omega$ and $R 2=1 \mathrm{M} \Omega$, since concern is given to achieve DC operating point stability. In order to reduce the computation time, application of $\mathrm{AI}$ technique has been introduced in this paper. The methodology 
used to maximize $V_{T H}$ and minimize transient time response is discussed in the following section.

\section{RESISTORS SELECTION BY USING GENETIC ALGORITHM (GA) AND ARTIFICIAL NEURAL NETWORK (ANN)}

In this Colpitts oscillator design, combination of Genetic Algorithm (GA) and Artificial Neural Network (ANN) is used in order to find optimize combination of $R 1$ and $R 2$ resistors those give highest Thevenin's equivalent voltage, $V_{T H}$ in order to maintain long term DC voltage stability, while attain shortest transient time response during start up period. GA plays the role to search for the optimize pair of $R 1$ and $R 2$ resistors values that will give maximum Thevenin's equivalent voltage $\left(V_{T H}\right)$. Thus, (3) can be rewriten in form of objective function for GA as

$$
f\left(R_{1}, R_{2}\right)=\frac{R_{2}}{R_{1}+R_{2}} V_{s}
$$

where $V_{s}$ is supply voltage, $R_{1}$ and $R_{2}$ is Thevenin's resistors. $V_{s}$ is selected to be $12 \mathrm{~V}$ DC voltage. The range of both resistors are set from $100 \mathrm{k} \Omega$ to $1 \mathrm{M} \Omega$ since simulation results (see APPENDIX A-E) show this range of resistors only able to produce pure sine wave output. The GA code is developed by using MATLAB. GA is used to search 500 optimize combination of resistors those can maximize the objective function. Thus, initial population is selected to be 500, while crossover and mutation rate are set as 0.25 and 0.1 respectively as initial variables. Thus, GA is expected to produce 500 optimize pair of resistor base on objective function as its output.

Then, one of the optimize resistor combinations among 500 optimize combinations will be selected by consider transient time response. In this stage, conventional method required 500 simulations to find transient response for transient response times for each pair of optimize resistor combinations generated by GA. In order to reduce the tedious job and computational time, ANN is used to learn the relationship between various resistors pair and transient time of the oscillator by using the data obtained from Table I. Once the ANN is converge (successfully learn the relationship), ANN is able to predict the transient response time for all 500 optimize resistor combinations generated by GA. The resistor combination of $R 1$ and $R 2$ that predicted by $\mathrm{ANN}$ that gives lowest transient response time will be used in order to achieve design objectives of the oscillator. The overall of the methodology is illustrated in the Fig. 6.

The Neural Network toolbox is used for the training and learning process. The developed BackPropagation (BP) ANN model consist of two input nodes, one hidden layer with 3 neurons and one neuron in output layer. Two input nodes and one output neuron are selected in order to train ANN with two values of $R_{1}$ and $R_{2}$ and one transient time for each resistor pair as output. Tansig activation function is used for all neurons. The data in the Table I are formatted by using unitary normalization as given by

$$
X_{\text {new }}=\frac{x}{x_{\max }}
$$



Fig. 6. Flow chart for optimized resistors selection

\section{RESULT AND DISCUSSION}

The GA is run to find the optimize pair of resistors $R 1$ and $R 2$ among the wide range of resistors values. The partial result of optimize pair of resistors given by GA is shown in the table II.

TABLE II.

PARTIAL LIST OF OPTIMIZED VALUE OF R1 AND R2 RESISTOR PAIRS

\begin{tabular}{|c|c|c|c|}
\hline $\mathbf{R 1}(\mathbf{k} \boldsymbol{\Omega})$ & $\mathbf{R 2}(\mathbf{k} \boldsymbol{\Omega})$ & $\mathbf{R} 1 \mathbf{( k \boldsymbol { \Omega } )}$ & $\mathbf{R 2}(\mathbf{k} \boldsymbol{\Omega})$ \\
\hline 100 & 979 & 100 & 979 \\
\hline 100 & 993 & 100 & 972 \\
\hline 100 & 993 & 100 & 993 \\
\hline 100 & 965 & 100 & 993 \\
\hline 100 & 979 & 100 & 958 \\
\hline 100 & 965 & 100 & 965 \\
\hline 100 & 965 & 100 & 979 \\
\hline 100 & 986 & 100 & 986 \\
\hline
\end{tabular}




\begin{tabular}{|c|c|c|c|}
\hline 100 & 910 & 100 & 1000 \\
\hline 100 & 1000 & 100 & 965 \\
\hline 100 & 979 & 100 & 979 \\
\hline
\end{tabular}

The list of optimized values of resistors pairs generated by GA as shown in the Table II are used as input data to ANN in order to predict the transient response time of for each pair of resistor. The transient time, $T$ predicted by using ANN are listed in the table III.

TABLE III.

TRANSIENT RESPONSE TIME (T) PREDICTED USING ARTIFICIAL NEURAL NETWORK FOR EACH PAIR OF OPTIMIZED VALUE OF RESISTORS

\begin{tabular}{|c|c|c|c|c|c|}
\hline $\mathbf{R} 1(\mathbf{k} \boldsymbol{\Omega})$ & $\mathbf{R 2}(\mathbf{k} \boldsymbol{\Omega})$ & $\mathbf{T}(\mathbf{m s})$ & $\mathbf{R 1}(\mathbf{k} \boldsymbol{\Omega})$ & $\mathbf{R 2}(\mathbf{k} \boldsymbol{\Omega})$ & $\mathbf{T}(\mathbf{m s})$ \\
\hline 100 & 979 & 1.29 & 100 & 979 & 1.29 \\
\hline 100 & 993 & 1.31 & 100 & 972 & 1.29 \\
\hline 100 & 993 & 1.31 & 100 & 993 & 1.31 \\
\hline 100 & 965 & 1.28 & 100 & 993 & 1.31 \\
\hline 100 & 979 & 1.29 & 100 & 958 & 1.27 \\
\hline 100 & 965 & 1.28 & 100 & 965 & 1.28 \\
\hline 100 & 965 & 1.28 & 100 & 979 & 1.29 \\
\hline 100 & 986 & 1.30 & 100 & 986 & 1.30 \\
\hline 100 & 910 & 1.22 & 100 & 1000 & 1.32 \\
\hline 100 & 1000 & 1.32 & 100 & 965 & 1.28 \\
\hline 100 & 979 & 1.29 & 100 & 979 & 1.29 \\
\hline
\end{tabular}

The result in the Table III shows that pair of $R 1=100 \mathrm{k} \Omega$ and $R 2=910 \mathrm{k} \Omega$ is expected to enter the steady state after $1.22 \mathrm{~ms}$ which is relatively faster than the rest of optimal combinations generated by GA. Thus, the aforementioned resistors combination are selected for proposed Colpitts oscillator design.

Fig. 7 shows the simulation of the Colpitts oscillator response with $R I=100 \mathrm{k} \Omega, R 2=910 \mathrm{k} \Omega$ and $R E=10 \mathrm{k} \Omega$. The values of inductor, $\mathrm{L}=0.796 \mathrm{mH}$ and capacitors, $C l=15$ $\mathrm{nF}$ and $C 2=680 \mathrm{nF}$ are selected randomly at this stage. Closer observation of the graph shows that the output enters steady state after $1.19 \mathrm{~ms}$. This result is very close compared to predicted transient time by using ANN.

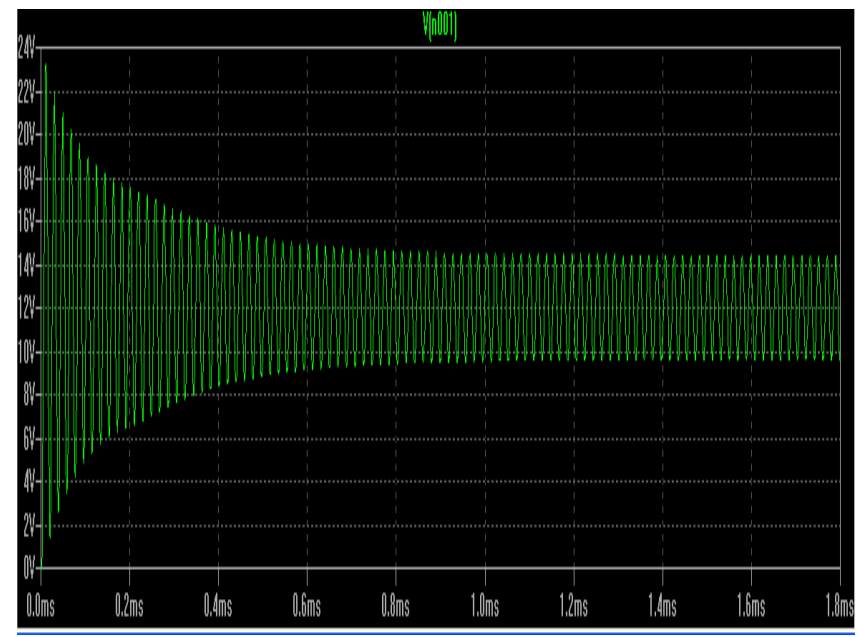

Fig. 7. Simulation of Colpitts oscillator waveform

\section{CONCLUSION}

In This works, utilization of Genetic Algorithm and Artificial Neural Network in common base Colpitts oscillator design has been suggested. This technique has been able to assist optimize selection of components values such as resistance values in the faster ways compared to conventional method. The designed common base Colpitts oscillator demonstrates optimization between transient time response and steady state response. This technique can be used for designing other complicated circuits as well.

\section{APPENDIX \\ OSCILLATOR OUTPUT ANALYSIS FOR VARIOUS RANGE OF R1 AND $R 2$}

All simulations are done by using capacitors with values $C 1=15 \mathrm{nF}$ and $C 2=680 \mathrm{nF}$, resistor $R E=10 \mathrm{k} \Omega$ and inductance $L 1=0.796 \mathrm{mH}$, while the values of resistance range $R 1$ and $R 2$ are changed from hundreds ohm to Mega ohm. The results from the simulation are shown from Fig. 8 to Fig. 12.

A. Analysis for $100 \Omega$ to $1 \mathrm{k} \Omega$ Range of $R 1$ and $R 2$ Resistors



Fig. 8. Output waveform for combination R1=100 $\Omega$ and $\mathrm{R} 2=500 \Omega$ combination resistors

Observation: Oscillator's output waveform fails to generate pure sine wave. 


\section{Resistors}

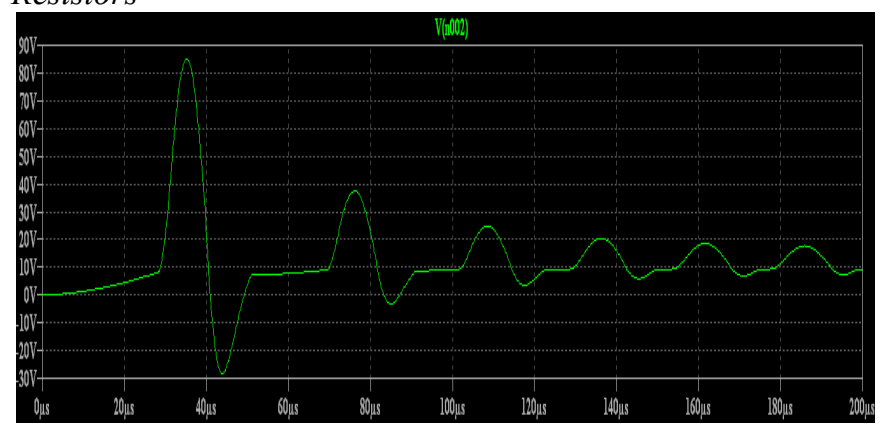

Fig. 9. Output waveform for combination $\mathrm{R} 1=1 \mathrm{k} \Omega$ and $\mathrm{R} 2=5 \mathrm{k} \Omega$ combination resistors

Observation: Output waveform fails to generate pure sine wave

C. Analysis for $10 \mathrm{k} \Omega$ to $100 \mathrm{k} \Omega$ range of $R 1$ and $R 2$

\section{Resistors}

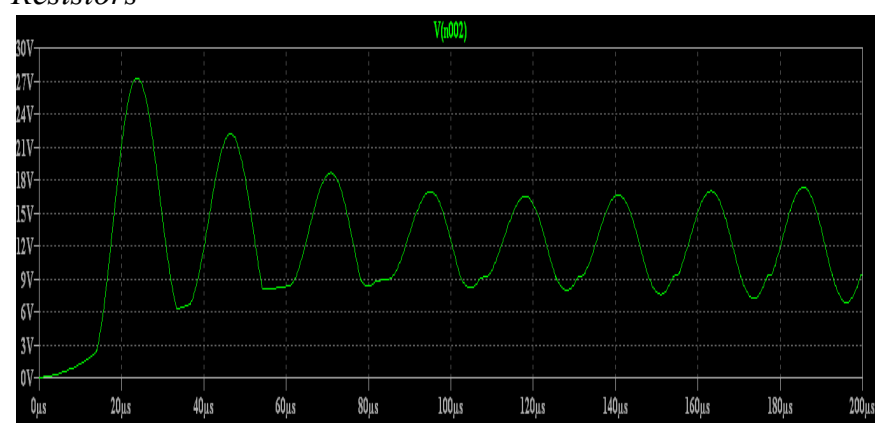

Fig. 10. Output waveform for combination $\mathrm{R} 1=10 \mathrm{k} \Omega$ and $\mathrm{R} 2=50 \mathrm{k} \Omega$ combination resistors

Observation: Oscillator not produces pure sine wave. Signal distortion and high overshoot occurred at beginning

D. Analysis for $100 \mathrm{k} \Omega$ to $1 \mathrm{M} \Omega$ range of $R 1$ and $R 2$ Resistors

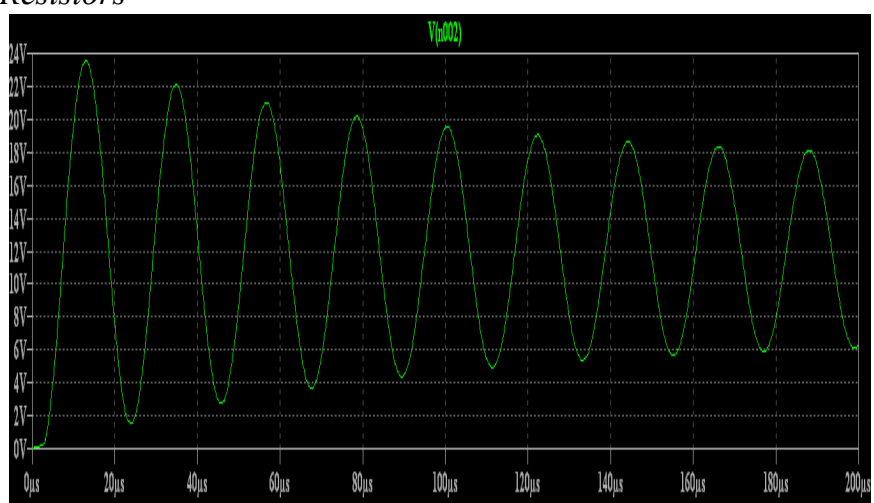

Fig. 11. Output waveform for combination $\mathrm{R} 1=100 \mathrm{k} \Omega$ and $\mathrm{R} 2=500 \mathrm{k} \Omega$ combination resistors

Observation: Combination of $\mathrm{R} 1=100 \mathrm{~K} \Omega$ and $\mathrm{R} 2=500 \mathrm{~K} \Omega$ successfully generate pure sine wave.
Resistors

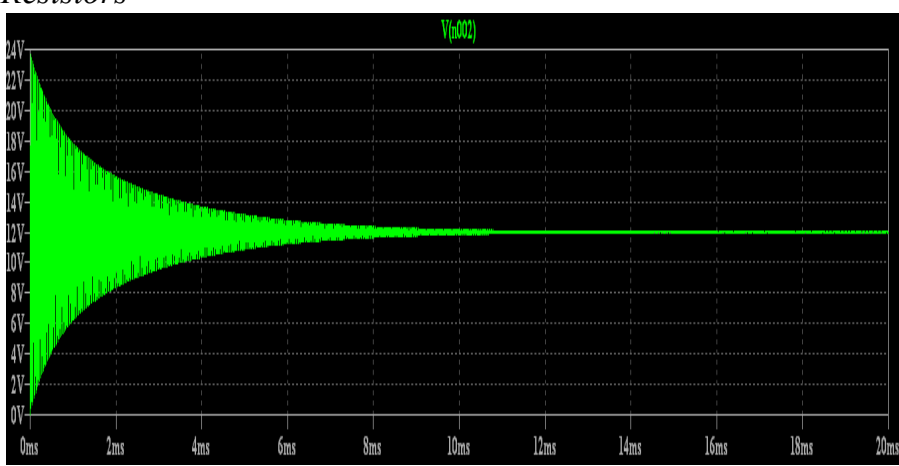

Fig. 12. Output waveform for combination $\mathrm{R} 1=1 \mathrm{M} \Omega$ and $\mathrm{R} 2=5 \mathrm{M} \Omega$ combination

Observation: Combination of $\mathrm{R} 1=1 \mathrm{M} \Omega$ and $\mathrm{R} 2=5 \mathrm{M} \Omega$ produce damped oscillation cause oscillate signal disappears after short period of time.

\section{REFERENCES}

[1] U. A. Bakshi and A. P. Godse. Analog Electronics. Pune, India: Technical Publication Pune, 2009.

[2] M. Fozdar, C. M. Arora and V. R. Gottipati. Recent Trends in Intelligent Techniques to Power Systems. In: UPEC, 2007, pp. 580-591.

[3] V. Aggarwal. Evolving sinusoidal oscillators using genetic algorithms. In: Proc. The 2003 NASA/DoD Conference on Evolvable Hardware, Chicago, USA, 2003, pp. 67-76.

[4] J. Zhang, Y. Shi, and Z. Zhan. Power Electronic Circuits Design: A Particle Swarm Optimization Approach. In: Proceedings of the $7^{\text {th }}$ International Conference on Simulated Evolution and Learning, 2008, pp. $605-614$

[5] P. Sen, R. J. Pratap, R. Mukhopadhyay, S. Sarkar, C. H. Lee, S. Pinel, G. S. May, and J. Laskar, Neuro-genetic design centering of millimeter wave oscillators. Silicon Monolithic Integrated Circuits in RF Systems, 2006, pp.4-5.

[6] L. Huelsbergen, E. Rietman and R. Slous. Evolving oscillators in Silicon. IEEE trans. on Evolutionary Computation, 1(3), 1999, pp. $197-$ 204

[7] R. O. Canham and A. M. Tyrrell. Evolved fault tolerance in evolvable hardware. In: Proceedings of the 2002 Congress on Evolutionary Computation, 2, 2002, pp. 1267-1271. 